\title{
Tiotropium add-on therapy reduces seasonal peaks of asthma worsening in adults with symptomatic severe asthma
}

\author{
To the Editor:
}

Despite the use of preferred controller therapies (including inhaled corticosteroids (ICS) with or without additional long-acting $\beta_{2}$-agonists (LABAs)), a large proportion of patients with asthma have poor disease control, leaving them at risk of recurring symptoms and episodes of asthma exacerbations and worsening $[1,2]$. Such problems can be triggered by many different environmental factors including pollutants, respiratory infections or allergens [3]. They may occur sporadically, but are often determined by the seasons, mirroring seasonal patterns of allergen exposure and prevalence of respiratory viral infection [3,4]. They can cause reduced quality of life, increase asthma burden [2,3], and have economic and societal impacts, such as increased absenteeism in school-age children, reduced work productivity and missed workdays in adults [5]. It is therefore important to choose an appropriate asthma treatment to achieve good symptom control and minimise the risk of exacerbations and worsening [2].

Tiotropium has been shown to be an efficacious add-on therapy to ICS in adult patients with symptomatic asthma across all severities [6-8]. In adults with symptomatic severe asthma, the PrimoTinA-asthma study consisted of two replicate phase III, randomised, double-blind, placebo-controlled, parallel-group, 48-week trials (NCT00772538/NCT00776984) [6]. Tiotropium $5 \mu \mathrm{g}$ add-on therapy prolonged the time to first asthma exacerbation (defined as an episode of progressive increase in one or more asthma symptom(s) (compared with usual day-to-day asthma symptoms), or a decline of $\geqslant 30 \%$ in morning peak expiratory flow $\left(\mathrm{PEF}_{\mathrm{am}}\right)$ for two or more consecutive days as judged by the investigator, requiring systemic corticosteroids (SCS) for $\geqslant 3$ days) during the 48 -week treatment period by 56 days, compared with placebo. This corresponded to a reduction in risk of $21 \%$ (hazard ratio (HR) $0.79,95 \%$ CI $0.62-1.00$; adjusted $\mathrm{p}=0.0343$ ).

In the same trials, data on asthma worsenings, a more moderate and common counterpart to exacerbations, were also collected (defined as an episode of progressive increase in one or more asthma symptom(s) (compared with usual day-to-day asthma symptoms) or a decline of $\geqslant 30 \%$ in $\mathrm{PEF}_{\mathrm{am}}$ for two or more consecutive days as judged by the investigator). Asthma worsenings include acute deteriorations not requiring SCS, in addition to those requiring SCS for $\geqslant 3$ days. Tiotropium add-on therapy was shown to increase the median time to first asthma worsening event by 134 days, corresponding to a reduction in risk of $31 \%$ (HR $0.69,95 \%$ CI $0.58-0.82$; p<0.0001) compared with placebo [6].

Here, we build on these results to explore whether the effects of tiotropium Respimat add-on therapy on episodes of asthma worsening in the PrimoTinA-asthma trials demonstrated any seasonality. The PrimoTinA-asthma trials were selected for this post hoc analysis as patients were recruited and commenced the 48-week treatment period across all months of the year. Episodes of asthma worsening were analysed as they were reported more frequently, capturing the full effect of tiotropium and reducing the likelihood of chance findings in rarer exacerbation events.

This was a post hoc analysis of pooled data (full analysis set $n=907$ ) from the two PrimoTinA-asthma trials, details of which have been reported previously [6]. The trials involved adults (aged 18-75 years) with symptomatic severe asthma despite at least daily high-dose ICS ( $\geqslant 800 \mu \mathrm{g}$ budesonide/equivalent) and LABA who were randomised to receive tiotropium $5 \mu \mathrm{g}$ or placebo via the Respimat. The number of

@ERSpublications

In adults with symptomatic severe asthma despite inhaled corticosteroid/long-acting $\beta_{2}$-agonist therapy, tiotropium add-on therapy reduces seasonal peaks of asthma worsening, providing a yearround benefit http://bit.ly/2m4LGuH

Cite this article as: FitzGerald JM, Buhl R, Casale TB, et al. Tiotropium add-on therapy reduces seasonal peaks of asthma worsening in adults with symptomatic severe asthma. Eur Respir J 2020; 55: 1900964 [https://doi.org/10.1183/13993003.00964-2019]. 
episodes of asthma worsening per month was analysed to determine any seasonality effect, with data from the Southern hemisphere shifted by 6 months (July=month 1; January=month 7) to align the seasonal time periods based on meteorological definitions [9].

The ratio of number of episodes of asthma worsening per patient was lower in patients receiving tiotropium versus placebo (rate ratio 0.76 , 95\% CI $0.63-0.91 ; \mathrm{p}=0.0031$ ). Moreover, when plotted by month, the number of episodes of asthma worsening in the placebo group showed a seasonal pattern, with peaks in autumn and winter months, and troughs in spring and summer months (figure 1). Tiotropium add-on treatment was shown to reduce the number of episodes of asthma worsening compared with placebo across all seasons, particularly during the autumn peak (figure 1). The seasonal pattern of episodes of asthma worsening in adults was similar to those reported in previous studies, with peaks in the autumn and winter months [10-12]. It has been suggested that seasonal patterns of asthma exacerbations and worsenings have an age-related difference in response to cyclic triggers [3], with adults more likely to experience peaks in autumn and winter months, and children more likely to experience exacerbations or worsenings in spring and autumn months $[11,12]$.

CASALE et al. [13] have previously reported that patients in the PrimoTinA-asthma trials were balanced between treatment groups at baseline in regard to potential allergic asthma (defined as serum immunoglobulin (Ig)E levels $>430 \mathrm{mg} \cdot \mathrm{L}^{-1}$, blood eosinophils $>0.6 \times 10^{9}$ cells $\cdot \mathrm{L}^{-1}$ and clinician judgement of asthma) (tiotropium versus placebo: serum $\operatorname{IgE}\left(>430 \mu \mathrm{g} \cdot \mathrm{L}^{-1}\right) \quad 45.4 \%$ versus $40.4 \%$; blood eosinophils $\left(>0.6 \times 10^{9}\right.$ cells $\left.\cdot \mathrm{L}^{-1}\right) 22.4 \%$ versus $19.1 \%$; clinician judgement of allergic asthma (yes) $60.5 \%$ versus $62.1 \%$; concomitant diagnosis of allergic rhinitis $22.6 \%$ versus $20.0 \%$ ). When categorical subgroup analyses were undertaken, it was demonstrated that tiotropium reduced the risk of both asthma exacerbations and worsening compared with placebo, independent of serum IgE levels, blood eosinophil counts and clinician judgement of allergic asthma.

The effect of tiotropium add-on to ICS and LABA on seasonal peaks is similar to that observed with other interventions, including ICS/LABA combinations and biologicals. SPAHN et al. [10] reported that dispensing of ICS/LABA during summer months was associated with a reduction in asthma-related emergency department visits or hospitalisations in the subsequent autumn compared with patients who did not receive ICS/LABA in patients aged 4-55 years with asthma. Busse et al. [14] reported that omalizumab almost eliminated spring and autumn exacerbation peaks in patients aged 6-20 years with persistent allergic asthma compared with placebo as add-on to ICS. A study of benralizumab provided further evidence of the beneficial effects of biologicals on seasonal peaks, with the authors reporting that benralizumab as add-on treatment to ICS/LABA in patients aged 12-75 years with severe uncontrolled eosinophilic asthma reduced, but did not eliminate, seasonal exacerbation peaks compared with placebo [11]. Furthermore, results from five tiotropium trials in paediatric patients with asthma and persistent asthmatic symptoms also demonstrate the efficacy of tiotropium add-on therapy in reducing seasonal peaks in adverse events related to asthma worsening and symptoms in children and adolescents (aged 1-17 years) [15]. Adverse events related to asthma worsening and symptoms were used as an alternative end-point because paediatric trials are often not sufficiently powered to assess asthma exacerbations and worsening.

FIGURE 1 Number of episodes of asthma worsening by season. Patients were recruited and commenced the 48-week treatment period across all months of the year.

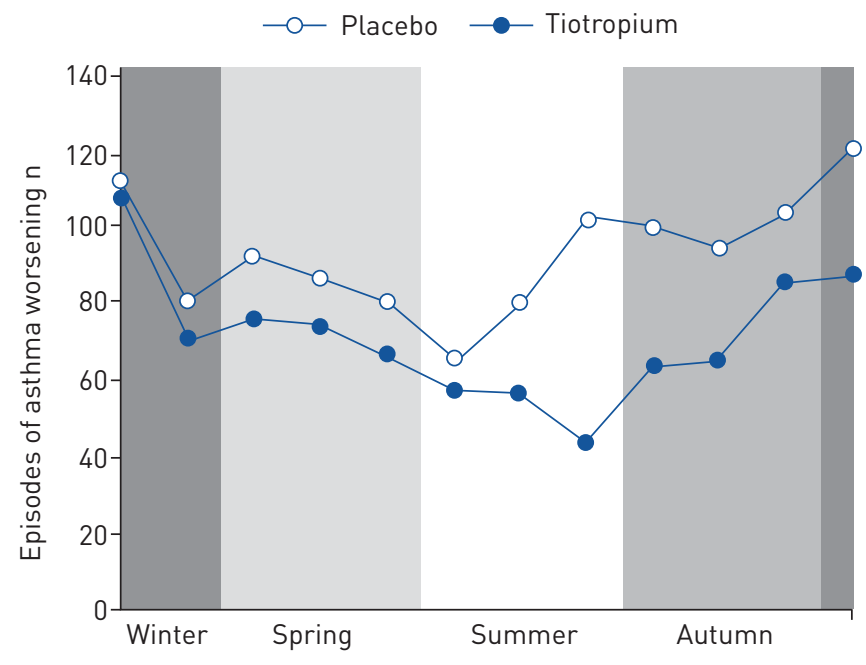


The strengths of this study include its duration, which was long enough to capture seasonal peaks, and the large population of patients with symptomatic severe asthma, details of which have been described previously [6]. Moreover, both trials were placebo-controlled and therefore provided the most valid comparison for assessing episodes of asthma worsening. However, one limitation of the study may include not capturing the cause of worsening within the trials. The narrow patient population (i.e. symptomatic severe asthma patients) may also be considered a limitation, but as this group is particularly prone to exacerbations and worsenings, it represents the optimal population to assess. Furthermore, despite proposals by groups including the Global Initiative for Asthma and the American Thoracic Society/ European Respiratory Society, there is no common consensus definition for asthma exacerbations or worsenings. It is suggested that there are rarely two studies that utilise the same definition, making comparisons across multiple clinical trials and studies complex [16].

This assessment of the effects of tiotropium in PrimoTinA-asthma broadens the evidence supporting the use of tiotropium as add-on therapy in patients with symptomatic asthma despite ICS/LABA therapy to reduce the risk of asthma exacerbations and worsening. This study enhances the literature showing that the peaks of episodes of asthma worsening follow a seasonal pattern. We have shown that tiotropium add-on treatment provides a year-round benefit to patients, which leads to reducing seasonal peaks of worsening.

J. Mark FitzGerald ${ }^{1}$, Roland Buhl ${ }^{2}$, Thomas B. Casale ${ }^{3}$, Branko Jugovic ${ }^{4}$, Liliana Zaremba-Pechmann ${ }^{5}$ and David M.G. Halpin ${ }^{6}$

${ }^{1}$ Centre for Heart and Lung Health, Vancouver, BC, Canada. ${ }^{2}$ Dept of Pulmonology, Johannes Gutenberg University Mainz, Mainz, Germany. ${ }^{3}$ Division of Allergy and Immunology, University of South Florida Morsani College of Medicine, Tampa, FL, USA. ${ }^{4}$ TA CardioMetabolism Respiratory Med, Boehringer Ingelheim International GmbH, Ingelheim am Rhein, Germany. ${ }^{5}$ Elderbrook Solutions $\mathrm{GmbH}$, Bietigheim-Bissingen, Germany. ${ }^{6}$ Royal Devon and Exeter Hospital, Exeter, UK.

Correspondence: J. Mark FitzGerald, Institute for Heart and Lung Health, 7th Floor, 2775 Laurel Street, Vancouver, V5Z 1M9, Canada. E-mail: Mark.Fitzgerald@vch.ca

Received: 13 May 2019 | Accepted after revision: 14 Sept 2019

Acknowledgements: The authors take full responsibility for the scope, direction, content and editorial decisions relating to the manuscript, were involved at all stages of development and have approved the submitted manuscript. Medical writing assistance, in the form of the preparation and revision of the draft manuscript, was supported financially by Boehringer Ingelheim and provided by Rosie Robson of MediTech Media (Manchester, UK), under the authors' conceptual direction and based on feedback from the authors.

Support statement: This work was funded by Boehringer Ingelheim. Funding information for this article has been deposited with the Crossref Funder Registry.

Conflict of interest: J.M. Fitzgerald reports grants from Boehringer Ingelheim, during the conduct of the study; grants and personal fees for lecturing and advisory board work from Boehringer Ingelheim, AstraZeneca, Novartis, Sanofi-Regeneron, Circassia and Teva; and grants from GlaxoSmithKline, outside the submitted work. R. Buhl reports grants to Mainz University and personal fees from Boehringer Ingelheim, GlaxoSmithKline, Novartis, and Roche, as well as personal fees from AstraZeneca, Chiesi, Cipla and Teva, outside the submitted work. T.B. Casale has undertaken consultancy work for AstraZeneca, Sanofi/Roche and Novartis, reports personal fees from and has undertaken consultancy work for Genentech, and reports grants from AstraZeneca, Sanofi/Roche, Genentech and Novartis, outside the submitted work. B. Jugovic is an employee of Boehringer Ingelheim. L. Zaremba-Pechmann was an external consultant for Boehringer Ingelheim at the time of the study. D.M.G. Halpin reports personal fees for lecturing, attending advisory boards and preparing educational materials from AstraZeneca, Chiesi and Pfizer, and grants for meeting attendance and personal fees for lecturing, attending advisory boards and preparing educational materials from Boehringer Ingelheim, GlaxoSmithKline and Novartis, outside the submitted work.

\section{References}

1 Peters SP, Ferguson G, Deniz Y, et al. Uncontrolled asthma: a review of the prevalence, disease burden and options for treatment. Respir Med 2006; 100: 1139-1151.

2 Global Initiative for Asthma. GINA Report: Global Strategy for Asthma Management and Prevention. 2018. https://ginasthma.org/wp-content/uploads/2019/01/2018-GINA.pdf Date last accessed: October 22, 2018.

3 Johnston NW, Sears MR. Asthma exacerbations. 1: epidemiology. Thorax 2006; 61: 722-728.

4 Staton TL, Arron JR, Olsson J, et al. Seasonal variability of severe asthma exacerbations and clinical benefit from lebrikizumab. J Allergy Clin Immunol 2017; 139: 1682-1684.e3.

5 Bahadori K, Doyle-Waters MM, Marra C, et al. Economic burden of asthma: a systematic review. BMC Pulm Med 2009; 9: 24.

6 Kerstjens HA, Engel M, Dahl R, et al. Tiotropium in asthma poorly controlled with standard combination therapy. N Engl J Med 2012; 367: 1198-1207.

7 Kerstjens HA, Casale TB, Bleecker ER, et al. Tiotropium or salmeterol as add-on therapy to inhaled corticosteroids for patients with moderate symptomatic asthma: two replicate, double-blind, placebo-controlled, parallel-group, active-comparator, randomised trials. Lancet Respir Med 2015; 3: 367-376. 
8 Paggiaro $\mathrm{P}$, Halpin DM, Buhl R, et al. The effect of tiotropium in symptomatic asthma despite low- to medium-dose inhaled corticosteroids: a randomized controlled trial. J Allergy Clin Immunol Pract 2016; 4: 104-113.e2.

9 Trenberth KE. What are the seasons? Bull Amer Meteor Soc 1983; 64: 1276-1282.

10 Spahn J, Sheth K, Yeh WS, et al. Dispensing of fluticasone propionate/salmeterol combination in the summer and asthma-related outcomes in the fall. J Allergy Clin Immunol 2009; 124: 1197-1203.

11 DuBuske L, Newbold $\mathrm{P}, \mathrm{Wu} \mathrm{Y}$, et al. Seasonal variability of exacerbations of severe, uncontrolled eosinophilic asthma and clinical benefits of benralizumab. Allergy Asthma Proc 2018; 39: 345-349.

12 Sears MR. Epidemiology of asthma exacerbations. J Allergy Clin Immunol 2008; 122: 662-668.

13 Casale TB, Tashkin DP, Luhmann R, et al. Tiotropium Respimat ${ }^{ø}$ add-on to at least ICS therapy demonstrates reduced risk of severe asthma exacerbation and asthma worsening in symptomatic asthma, independent of IgE or blood eosinophil levels. J Allergy Clin Immunol 2016; 137: AB214.

14 Busse WW, Morgan WJ, Gergen PJ, et al. Randomized trial of omalizumab (anti-IgE) for asthma in inner-city children. N Engl J Med 2011; 364: 1005-1015.

15 Vogelberg C, Szefler SJ, Vrijlandt EJLE, et al. Tiotropium add-on therapy is safe and reduces seasonal worsenings in paediatric asthma patients. Eur Respir J 2019; 53: 1801824.

16 Fuhlbrigge A, Peden D, Apter AJ, et al. Asthma outcomes: exacerbations. J Allergy Clin Immunol 2012; 129: S34-S48. 